\title{
Efeito da Temperatura nas CaRacterísticas Físico-Químicas de Soluções Aquosas com AdJuvantes de Uso AgRícola ${ }^{1}$
}

\author{
Temperature Effect on the Physical-Chemical Characteristics of Aqueous Solutions with Spray \\ Adjuvants
}

\author{
CUNHA, J.P.A.R. ${ }^{2}$, ALVES, G.S. ${ }^{3}$ e REIS, E.F. ${ }^{4}$
}

\begin{abstract}
RESUMO - A eficácia de tratamentos fitossanitários depende das características físicoquímicas das caldas de pulverização. Dessa forma, o presente trabalho teve como objetivo avaliar o efeito da temperatura da calda e da adição de adjuvantes de uso agrícola nas características físico-químicas de soluções aquosas para aplicação de agrotóxicos. Foram avaliados potencial hidrogeniônico, condutividade elétrica, densidade, viscosidade dinâmica, tensão superficial e estabilidade de soluções aquosas contendo quatro adjuvantes comerciais de uso agrícola, além de uma amostra com apenas água, submetidas a temperaturas de $5{ }^{\circ} \mathrm{C}$, $15^{\circ} \mathrm{C}$ e $25^{\circ} \mathrm{C}$. Foi utilizado delineamento inteiramente casualizado, com quatro repetições, em fatorial 5 x 3. De acordo com os resultados, pôde-se concluir que a alteração da temperatura da calda influenciou as características físico-químicas de maneira diferenciada para cada adjuvante, o que demonstra a complexidade do estudo dessa relação e a impossibilidade de fazer generalizações. O efeito dos adjuvantes nas caracterísiticas fisico-químicas das soluções aquosas mostrou-se dependente de sua composição química e formulação. A temperatura influenciou a viscosidade com maior intensidade, enquanto a tensão superficial foi a caracteristica físico-química mais sensivel ao uso de adjuvante.
\end{abstract}

Palavras-chave: aditivos de calda, agroquímicos, surfatantes, tecnologia de aplicação.

\begin{abstract}
Pesticide application efficiency is dependent upon the physical-chemical characteristics of the spray aqueous solution. Thus, this study evaluated adjuvant addition and temperature effect on the physical-chemical characteristics of pesticide spray aqueous solutions. Hydrogenionic potential, electrical conductivity, density, dynamic viscosity, surface tension and stability of aqueous solution with four commercial adjuvants were evaluated, besides water alone. A randomized design with four replications was used, in a factorial scheme (5 $x$ 3). According to the results, it could be concluded that the temperature effect on the physical-chemical characteristics was different for each adjuvant, showing that no simple or linear relationship could be observed. The adjuvant effect on the physicalchemical characteristics of aqueous solutions was based on their chemical properties and formulation. Temperature affected viscosity with more intensity, while surface tension was the most sensitive characteristic of adjuvant addition.
\end{abstract}

Keywords: spray additives, agrochemicals, surfactants, application technology.

\section{INTRODUÇÃO}

O processo de subdivisão do líquido em gotas, conhecido como pulverização, é a base da tecnologia de aplicação de agrotóxicos. Dele dependem o potencial de deriva, a perda por escorrimento e a cobertura do alvo. Trata-se de um processo complexo, influenciado por diversos fatores, como o tipo de equipamento de pulverização empregado e as propriedades físico-químicas da calda (Cunha et al., 2003; Broniarz-Press et al., 2009).

1 Recebido para publicação em 6.10.2009 e na forma revisada em 3.9.2010.

2 Professor, Dr., Instituto de Ciências Agrárias, Universidade Federal de Uberlândia - ICIAG/UFU, 38400-902 Uberlândia-MG, <jpcunha@iciag.ufu.br>; ${ }^{3}$ Acadêmico de Agronomia, ICIAG/UFU; ${ }^{4}$ Professor, Dr., Universidade Estadual de Goiás - UEG, 75220-400 Anápolis-GO. 
Vários estudos já foram realizados para caracterizar o espectro de gotas em função dos equipamentos de pulverização (Cunha et al., 2007; Nuyttens et al., 2007). Contudo, pouca informação existe a respeito das propriedades físico-químicas das caldas e dos fatores que as influenciam, provavelmente devido à sua complexidade (Schampheleire et al., 2008). Downer et al. (1998), estudando o processo de pulverização em diferentes caldas, também mostram que muitos fatores o influenciam, dificultando o estabelecimento de relações simples.

A formação das gotas e a ação dos agrotóxicos são dependentes de constituintes da calda de pulverização, que, embora não compondo o ingrediente ativo, podem melhorar sua eficácia (Green \& Beestman, 2007). Ramsdale \& Messersmith (2001) afirmam que os adjuvantes podem melhorar a eficácia das aplicações, porém a interação adjuvante e agrotóxico é um processo complexo, que envolve muitos aspectos físicos, químicos e fisiológicos, e pode variar para cada condição testada. Os adjuvantes atuam de maneira diferente entre si e podem promover melhoras no molhamento, na aderência, no espalhamento, na redução de espuma e na dispersão da calda de pulverização por meio da alteração das propriedades físico-químicas da calda (Montório et al., 2004; Carbonari et al., 2005; Costa et al., 2005; Mendonça et al., 2007). A adição de adjuvantes pode alterar o desempenho das aplicações; contudo, seu efeito pode ser positivo ou até mesmo negativo no que se refere à deposição do produto no alvo.

O grau de pulverização, incluindo-se aí o diâmetro da mediana volumétrica, está ligado à viscosidade e à tensão superficial da calda. Em geral, a elevação da viscosidade está associada à geração de gotas de pulverização maiores e, portanto, com efeito no potencial de deriva de uma aplicação (Schampheleire et al., 2008). No entanto, não há definida a magnitude dessa elevação necessária para o aumento do diâmetro das gotas. Bouse et al. (1990) mostram que os componentes das formulações são importantes na determinação das características da pulverização, como o tamanho de gota. Entretanto, a variação da viscosidade proporcionada pela adição de adjuvantes não é muito significativa.
Segundo Montório et al. (2005), o acréscimo de surfatantes nas caldas aquosas de pulverização contribui para a formação de filmes líquidos sobre as superficies foliares, graças ao processo de coalescência das gotas. Uma menor tensão superficial permite, ainda, transpor obstáculos como a presença de pelos foliares; com isso, aumenta a quantidade de princípio ativo que atinge as áreas de absorção (Martins et al., 2005).

Além dos adjuvantes, a temperatura da calda também pode influenciar as propriedades físico-químicas e, consequentemente, o processo de pulverização. Variações de $0{ }^{\circ} \mathrm{C}$ a $30{ }^{\circ} \mathrm{C}$ na calda são comuns nas aplicações em campo, dependendo da região e do horário de aplicação. Teoricamente, são esperadas mudanças nas características da calda, porém se desconhece a magnitude e a interação com os componentes da mistura. Além disso, a redução da temperatura da calda pode ser uma estratégia para redução da evaporação, a ser utilizada por fabricantes de pulverizadores com o emprego, por exemplo, de tubos de vórtices, que são dispositivos mecânicos simples e de baixo custo utilizados para refrigeração (Contreras et al., 2008). Isso demonstra a importância de se conhecer o comportamento da calda submetida a diferentes temperaturas.

O presente trabalho teve como objetivo avaliar o efeito da temperatura da calda e da adição de adjuvantes de uso agrícola nas características físico-químicas de soluções aquosas para aplicação de agrotóxicos.

\section{MATERIAL E MÉTODOS}

O presente trabalho foi realizado no Laboratório de Mecanização Agrícola da Universidade Federal de Uberlândia. Foram avaliados potencial hidrogeniônico, condutividade elétrica, densidade, viscosidade, tensão superficial e estabilidade de soluções aquosas em diferentes temperaturas, contendo quatro adjuvantes comerciais de uso agrícola, em sua dose recomendada pelo fabricante, além de uma amostra com apenas água destilada. As temperaturas testadas foram de $5{ }^{\circ} \mathrm{C}, 15{ }^{\circ} \mathrm{C}$ e $25^{\circ} \mathrm{C}$. Os adjuvantes e suas formulações e as doses avaliadas estão apresentados na Tabela 1. Vale destacar que os fabricantes não disponibilizam nas bulas detalhes sobre a 
Tabela 1 - Adjuvantes e doses avaliadas

\begin{tabular}{|c|c|c|c|}
\hline Composição básica do adjuvante & Indicação de uso & Formulação & $\begin{array}{c}\text { Dose } \\
(\% \mathrm{v} / \mathrm{v})\end{array}$ \\
\hline $\begin{array}{l}\text { Éster metílico de óleo de soja }\left(720 \mathrm{~g} \mathrm{~L}^{-1}\right) \text {. Produto } \\
\text { comercial: Áureo } \AA \text {. }\end{array}$ & Espalhante adesivo & Concentrado emulsionável & 0,25 \\
\hline $\begin{array}{l}\text { Copolímero poliéter polimetil siloxano }\left(750 \mathrm{~g} \mathrm{~L}^{-1}\right) \text {. } \\
\text { Produto comercial: Break Thru®. }\end{array}$ & Espalhante adesivo & Concentrado solúvel & 0,10 \\
\hline $\begin{array}{l}\text { Ésteres de ácidos graxos com glicol }\left(930 \mathrm{~mL} \mathrm{~L}^{-1}\right) \text {. } \\
\text { Produto Comercial: Natur'óleo®. }\end{array}$ & Espalhante adesivo & Emulsão óleo em água & 0,50 \\
\hline $\begin{array}{l}\text { Óleo mineral - hidrocarbonetos alifáticos }\left(428 \mathrm{~g} \mathrm{~L}^{-1}\right) \text {. } \\
\text { Produto comercial: Nimbus }{ }^{\circledR} \text {. }\end{array}$ & Espalhante adesivo & Concentrado emulsionável & 0,50 \\
\hline
\end{tabular}

formulação, como a concentração de emulsificante, o que poderia facilitar a compreensão sobre sua forma de ação. Foi utilizado delineamento inteiramente casualizado, com quatro repetições, num fatorial $5 \times 3$ (cinco composições de calda e três temperaturas).

Todas as avaliações foram realizadas a partir de soluções preparadas em béqueres de $0,5 \mathrm{~L}$, com temperatura controlada por termômetro digital com resolução de $0,1{ }^{\circ} \mathrm{C}$ e precisão de $\pm 0,4{ }^{\circ} \mathrm{C}$. $\mathrm{O} \mathrm{pH}$ e a condutividade elétrica foram medidos diretamente nas soluções, utilizando peagômetro e condutivímetro portátil (Hanna, HI98139). O equipamento foi previamente calibrado por meio de soluçõespadrão e compensação automática de temperatura desativada. A densidade foi estimada por meio da determinação da massa de $0,1 \mathrm{~L}$ da solução depositada em um balão volumétrico, em balança com resolução de $0,1 \mathrm{mg}$.

A viscosidade dinâmica foi determinada empregando-se um viscosímetro rotativo microprocessado (Quimis, Q860M21), o qual permite medir eletronicamente a força de torção já convertida em viscosidade. Esse viscosímetro de medição direta funciona pelo princípio da rotação de um cilindro (cabeça de medição) submerso na amostra a ser analisada, medindo-se a força da torção necessária para superar a resistência da rotação. Utilizou-se o rotor zero e rotação de $60 \mathrm{rpm}$.

A tensão superficial foi determinada por um tensiômetro de bancada com anel de platina (Kruss, K6), empregando o método Du Nouy (Dopierala \& Prochaska, 2008). O teste consiste em medir a tensão sofrida pelo anel que fica na extremidade de uma haste flexivel, colocada sobre a superficie da amostra e pressionada contra esta até que sofra repulsão. O tensiômetro foi calibrado com água destilada.

A estabilidade da calda foi avaliada de forma visual. Após o preparo das soluções e descanso por uma hora, elas foram agitadas homogeneamente por 60 segundos. Posteriormente, após 10, 20, 40, 60 e 120 minutos, verificou-se a formação de fases na solução: estável (monofásica) e instável (bifásica). Avaliou-se também a formação de espuma na solução.

Os dados de $\mathrm{pH}$, condutividade elétrica, densidade, viscosidade e tensão superficial foram submetidos à análise de variância, e as médias, comparadas entre si, utilizando-se o teste de Scott-Knott a 5\% de probabilidade.

\section{RESULTADOS E DISCUSSÃO}

A análise de variância mostrou que em todas as propriedades analisadas, com exceção da densidade, houve interação significativa entre o adjuvante e a temperatura, indicando a dependência entre os fatores. Percebe-se que não é possivel fazer generalizações quanto ao efeito da temperatura e da adição de adjuvante à calda no que diz respeito às propriedades físico-químicas. Downer et al. (1998) também mostraram que o efeito da temperatura e de adjuvantes na pulverização é um processo complexo, que dificulta o estabelecimento de relações claras e diretas.

$\mathrm{O} \mathrm{pH}$ e a condutividade elétrica das soluções são mostrados na Tabela 2. $\mathrm{O}$ pH, em média, variou de 6,45 a 7,06 , demonstrando pequena amplitude de variação em termos 
práticos. Green \& Beestman (2007) afirmam que a ação dos adjuvantes sobre o pH é bastante variada, podendo levar ao seu aumento, redução ou inalteração. A redução da temperatura levou ao aumento do $\mathrm{pH}$. A temperatura altera o equilíbrio de dissociação da água, facilitando a quebra da ligação entre oxigênio e hidrogênio. $O$ aumento da temperatura aumenta a atividade dos ions hidrogênio e desloca o equilíbrio químico da água, tornando-a mais ácida, porém o efeito não foi muito pronunciado.

Segundo Azevedo (2001), os produtos são formulados para tolerar alguma variabilidade no $\mathrm{pH}$ das caldas. Valores extremos, no entanto, podem alterar a estabilidade física. Dessa forma, é importante consultar o fabricante para verificar a faixa de $\mathrm{pH}$ ideal para cada agrotóxico e, assim, determinar o adjuvante mais adequado.

Esses resultados alertam para os cuidados que devem ser tomados na utilização dessas soluções para a aplicação de agrotóxicos, uma vez que a eficiência de alguns destes, como é o caso dos grupamentos ácidos do 2,4-D e do glifosate, é dependente do $\mathrm{pH}$ da calda (Wanamarta \& Penner, 1989).

Alguns herbicidas têm sua eficiência elevada na planta com a redução do $\mathrm{pH}$ da água a valores próximos a 4,0. Além disso, em $\mathrm{pH}$ mais baixo, a taxa de hidrólise é retardada, mantendo a folha úmida por maior tempo, pois a superficie das folhas, em geral, tem $\mathrm{pH}$ neutro, havendo interação com o $\mathrm{pH}$ da calda.
Com relação à condutividade elétrica, os produtos copolimero poliéter polimetil, ésteres de ácidos graxos com glicol e o óleo mineral reduziram a condutividade, enquanto o éster metílico de óleo de soja promoveu grande aumento. Nota-se que os dois produtos derivados de óleo vegetal apresentaram comportamento diferente, o que pode ser explicado pela diferença na formulação (Cunha \& Carvalho, 2005). A redução da temperatura levou à redução da condutividade elétrica.

De acordo com Carlson \& Burnside (1984), a condutividade elétrica da água, quando elevada, indica a presença de grande quantidade de íns, os quais podem diminuir a eficácia biológica de alguns herbicidas. Íons como $\mathrm{Fe}^{+3} \mathrm{e} \mathrm{Al}^{+3}$, por exemplo, podem reagir com o agrotóxico, reduzindo sua eficácia. No entanto, essa interferência é influenciada pela tecnologia empregada na pulverização, não existindo uma concentração alta ou baixa fixa para tais íons. Para uma mesma concentração, quanto menor o volume de água utilizado por área para a distribuição de uma mesma dose de agrotóxico, menor será a interferência deste sobre o princípio ativo (Ramos \& Araújo, 2006).

Na Tabela 3 são apresentados os resultados de densidade e viscosidade. A adição dos adjuvantes não promoveu alteração da densidade, concordando com os dados apresentados por Matuo et al. (1989). A variação da temperatura também não foi suficiente para promover diferenciação significativa na densidade.

Tabela 2 - Potencial hidrogeniônico e condutividade elétrica $\left(\mu \mathrm{S} \mathrm{cm}^{-1}\right)$ de soluções aquosas em três temperaturas, contendo diferentes adjuvantes

\begin{tabular}{|c|c|c|c|c|c|c|c|c|}
\hline \multirow{2}{*}{ Adjuvante } & \multicolumn{4}{|c|}{$\overline{\mathrm{pH}}$} & \multicolumn{4}{|c|}{ Condutividade elétrica $\left(\mu \mathrm{S} \mathrm{cm}^{-1}\right)$} \\
\hline & $5{ }^{\circ} \mathrm{C}$ & $15^{\circ} \mathrm{C}$ & $25^{\circ} \mathrm{C}$ & Média & $5^{\circ} \mathrm{C}$ & $15^{\circ} \mathrm{C}$ & $25^{\circ} \mathrm{C}$ & Média \\
\hline Éster metílico de óleo de soja & $6,87 \mathrm{bA}$ & $6,81 \mathrm{bB}$ & $6,76 \mathrm{aB}$ & 6,81 & $13,92 \mathrm{aC}$ & $16,91 \mathrm{aB}$ & $23,00 \mathrm{aA}$ & 17,94 \\
\hline Copolímero poliéter polimetil & $7,06 \mathrm{aA}$ & $6,88 \mathrm{aB}$ & $6,45 \mathrm{cC}$ & 6,80 & $5,45 \mathrm{cB}$ & $6,50 \mathrm{cB}$ & $8,19 \mathrm{dA}$ & 6,71 \\
\hline Ésteres de ácidos graxos com glicol & $6,90 \mathrm{bA}$ & $6,92 \mathrm{aA}$ & $6,77 \mathrm{aB}$ & 6,86 & $5,64 \mathrm{cB}$ & $5,84 \mathrm{cB}$ & $9,10 \mathrm{dA}$ & 6,86 \\
\hline Óleo mineral & $7,04 \mathrm{aA}$ & $6,93 \mathrm{aB}$ & $6,68 \mathrm{bC}$ & 6,88 & $5,18 \mathrm{cC}$ & $6,41 \mathrm{cB}$ & $10,84 \mathrm{cA}$ & 7,48 \\
\hline Água & $6,87 \mathrm{bA}$ & $6,79 \mathrm{bA}$ & $6,52 \mathrm{cB}$ & & $6,98 \mathrm{bC}$ & $9,31 \mathrm{bB}$ & $12,91 \mathrm{bA}$ & 9,73 \\
\hline Média & 6,97 & 6,89 & 6,67 & & 7,43 & 8,99 & 12,81 & \\
\hline CV (\%) & \multicolumn{4}{|c|}{0,82} & \multicolumn{4}{|c|}{7,51} \\
\hline
\end{tabular}

Médias seguidas por letras distintas maiúsculas, nas linhas, e minúsculas, nas colunas, diferem significativamente entre si a $5 \%$ de probabilidade pelo teste de Scott-Knott. 
Tabela 3 - Densidade $\left(\mathrm{g} \mathrm{cm}^{-3}\right)$ e viscosidade ( $\mathrm{mPa}$ s) de soluções aquosas em três temperaturas, contendo diferentes adjuvantes

\begin{tabular}{|c|c|c|c|c|c|c|c|c|}
\hline \multirow{2}{*}{ Adjuvante } & \multicolumn{4}{|c|}{ Densidade $\left(\mathrm{g} \mathrm{cm}^{-3}\right)$} & \multicolumn{4}{|c|}{ Viscosidade (mPa s) } \\
\hline & $5^{\circ} \mathrm{C}$ & $15^{\circ} \mathrm{C}$ & $25^{\circ} \mathrm{C}$ & Média & $5^{\circ} \mathrm{C}$ & $15^{\circ} \mathrm{C}$ & $25^{\circ} \mathrm{C}$ & Média \\
\hline Éster metílico de óleo de soja & 0,9886 & 0,9902 & 0,9867 & $0,9885 \mathrm{a}$ & $1,88 \mathrm{aA}$ & $1,39 \mathrm{aB}$ & $1,04 \mathrm{bC}$ & 1,44 \\
\hline Copolímero poliéter polimetil & 0,9915 & 0,9950 & 0,9896 & $0,9920 \mathrm{a}$ & $1,89 \mathrm{aA}$ & $1,33 \mathrm{aB}$ & $1,09 \mathrm{aC}$ & 1,44 \\
\hline Ésteres de ácidos graxos com glicol & 0,9884 & 0,9936 & 0,9890 & $0,9903 \mathrm{a}$ & $1,92 \mathrm{aA}$ & $1,38 \mathrm{aB}$ & $1,11 \mathrm{aC}$ & 1,44 \\
\hline Óleo mineral & 0,9909 & 0,9889 & 0,9937 & $0,9912 \mathrm{a}$ & $1,81 \mathrm{aA}$ & $1,36 \mathrm{aB}$ & $1,03 \mathrm{bC}$ & 1,40 \\
\hline Água & 0,9881 & 0,9917 & 0,9923 & $0,9907 \mathrm{a}$ & $1,63 \mathrm{bA}$ & $1,25 \mathrm{bB}$ & $1,00 \mathrm{bC}$ & 1,29 \\
\hline Média & $0,9895 \mathrm{~A}$ & $0,9919 \mathrm{~A}$ & $0,9903 \mathrm{~A}$ & & 1,83 & 1,32 & 1,05 & \\
\hline CV $(\%)$ & \multicolumn{4}{|c|}{0,46} & \multicolumn{4}{|c|}{4,96} \\
\hline
\end{tabular}

Médias seguidas por letras distintas maiúsculas, nas linhas, e minúsculas, nas colunas, diferem significativamente entre si a $5 \%$ de probabilidade pelo teste de Scott-Knott.

Apesar de a densidade ser uma propriedade cujo aumento pode levar à redução de deriva, nota-se que na prática esse processo não é facilmente obtido, principalmente pela baixa concentração de adjuvantes em relação à água. Para se conseguir aumento de densidade, seria necessário aumentar muito a concentração, o que oneraria bastante a aplicação.

Todos os produtos elevaram a viscosidade das soluções em relação à água, nas temperaturas de 5 e $15{ }^{\circ} \mathrm{C}$. Na temperatura de $25^{\circ} \mathrm{C}$, a dose adicionada do óleo mineral e do éster metílico de óleo de soja não foi suficiente para alterar a viscosidade. Downer et al. (1998), avaliando a viscosidade de calda com diversos adjuvantes, também notaram que alguns produtos promovem ligeiro aumento de viscosidade, enquanto outros têm comportamento semelhante ao da água.

$\mathrm{O}$ aumento da temperatura promoveu a redução da viscosidade em todas as soluções testadas. A resistência de um fluido ao cisalhamento depende da coesão. Em um líquido, cujas moléculas estão muito mais próximas que num gás, existem forças de coesão muito maiores que nos gases. A coesão parece ser a causa predominante da viscosidade num líquido; como ela diminui com a temperatura, a viscosidade segue o mesmo comportamento.

Smith et al. (2000), estudando a deposição de herbicida em folhas de capim-fedegoso (Cassia occidentalis), não encontraram diferença entre soluções sem e com a adição de adjuvante para aumento da viscosidade da calda (polímero de acrilamida). Neste trabalho, a viscosidade das soluções foi medida, tendo variado de $1,1 \mathrm{mPa} \mathbf{s}$, sem adjuvante, para $8,9 \mathrm{mPa}$ s, com adjuvante, o que mostra que variações dessa ordem podem não interferir na deposição da calda no alvo.

No tocante à tensão superficial (Tabela 4), todos os adjuvantes promoveram a sua redução em relação à água. $\mathrm{O}$ adjuvante copolímero poliéter polimetil foi o mais eficiente nessa redução, concordando com os dados apresentados por Montório et al. (2005), em que esse produto também foi bom redutor de tensão superficial.

A temperatura teve comportamento diferenciado para cada produto. Para o copolímero poliéter polimetil e para os ésteres de ácidos graxos com glicol, ela não alterou a tensão superficial, enquanto para os demais, inclusive a água, o resfriamento levou ao aumento da tensão superficial. O gradiente máximo de tensão obtido com a variação de temperatura foi de $0,0071 \mathrm{~N} \mathrm{~m}^{-1}$, para o éster metílico de óleo de soja.

Singh \& Mack (1993), avaliando tensão superficial, observaram que os surfatantes organossiliconados estudados apresentaram valores mínimos de $0,0225 \mathrm{~N} \mathrm{~m}^{-1}$, em concentrações que variavam de 0,1 a $0,125 \%$. Quando estudaram espalhantes não siliconados, pertencentes ao grupo do alquilfenol etoxilado, verificaram tensão de equilíbrio próxima a $0,0310 \mathrm{~N} \mathrm{~m}^{-1}$, em concentrações nos mesmos níveis observados nos siliconados.

A pequena área de contato entre a gotícula da calda pulverizada e a superficie da cutícula 
limita o potencial para a difusão da calda; logo, todos os aspectos das superfícies foliares (topografia das células, grau e tipo de desenvolvimento da cera epicuticular, tricomas e glândulas) também influenciam a deposição do agrotóxico nas superfícies foliares, além da tensão superficial (Albert \& Victoria Filho, 2002).

Os dados obtidos de estabilidade das caldas são apresentados na Tabela 5 . Os sinais positivos $(+)$ indicam estabilidade da calda no referido tempo, e os sinais negativos (-) representam instabilidade da calda. Nota-se que o produto ésteres de ácidos graxos com glicol mostrou-se instável após $10 \mathrm{~min}$ sem agitação. Não houve interferência da temperatura nos resultados. Em nenhuma solução foi notada a formação de espuma, com exceção da solução com o produto copolímero poliéter polimetil, nos tempos observados. Especial cuidado deve ser dado ao sistema de agitação de calda do tanque dos pulverizadores em soluções que apresentam baixa estabilidade.
Tendo em vista os resultados apresentados, a adição de adjuvantes às caldas de pulverização pode ser importante quando da aplicação de diversos agrotóxicos, porém não deve ser uma prática generalizada, tendo em vista seu comportamento distinto em cada tipo de aplicação. Essa constatação reforça as conclusões de Downer et al. (1998). Esses autores mostram que o estudo do efeito de adjuvantes na calda de pulverização é específico para cada produto e formulação empregada.

Segundo Ryckaert et al. (2007), o uso correto dos adjuvantes pode aumentar significativamente o desempenho dos produtos aplicados. Contudo, o aumento na eficiência da aplicação do agrotóxico pode causar aumento do impacto ambiental. Isso é possivel pela presença da molécula do adjuvante no ambiente e também pela influência do adjuvante no resíduo final do agrotóxico. Com o emprego dos adjuvantes, os períodos de carência devem ser reestudados, em função do aumento dos resíduos dos produtos nos vegetais.

Tabela 4 - Tensão superficial $\left(\mathrm{N} \mathrm{m}^{-1}\right)$ de soluções aquosas em três temperaturas, contendo diferentes adjuvantes

\begin{tabular}{|l|c|c|c|c|}
\hline \multirow{2}{*}{ Adjuvante } & \multicolumn{4}{c|}{ Tensão superficial $\left(\mathrm{N} \mathrm{m}^{-1}\right)$} \\
\cline { 2 - 5 } & $5{ }^{\circ} \mathrm{C}$ & $15^{\circ} \mathrm{C}$ & $25{ }^{\circ} \mathrm{C}$ & Média \\
\hline Éster metílico de óleo de soja & $0,0397 \mathrm{bA}$ & $0,0372 \mathrm{bB}$ & $0,0326 \mathrm{cC}$ & 0,0365 \\
\hline Copolímero poliéter polimetil & $0,0278 \mathrm{dA}$ & $0,0272 \mathrm{cA}$ & $0,0266 \mathrm{dA}$ & 0,0272 \\
\hline Ésteres de ácidos graxos com glicol & $0,0378 \mathrm{cA}$ & $0,0376 \mathrm{bA}$ & $0,0370 \mathrm{bA}$ & 0,0375 \\
\hline Óleo mineral & $0,0368 \mathrm{cA}$ & $0,0364 \mathrm{bA}$ & $0,0356 \mathrm{bA}$ & 0,0363 \\
\hline Água & $0,0764 \mathrm{aA}$ & $0,0747 \mathrm{aA}$ & $0,0716 \mathrm{aB}$ & 0,0742 \\
\hline \multicolumn{1}{|c|}{ Média } & 0,0437 & 0,0426 & 0,0407 & \\
\hline \multicolumn{1}{|c|}{$\mathrm{CV}(\%)$} & & & 2,21 & \\
\hline
\end{tabular}

Médias seguidas por letras distintas maiúsculas, nas linhas, e minúsculas, nas colunas, diferem significativamente entre si a $5 \%$ de probabilidade pelo teste de Scott-Knott.

Tabela 5 - Estabilidade de soluções aquosas em três temperaturas, contendo diferentes adjuvantes

\begin{tabular}{|c|c|c|c|c|c|c|c|c|c|c|c|c|c|c|c|}
\hline \multirow{3}{*}{ Adjuvante } & \multicolumn{5}{|c|}{ Estabilidade $\left(5^{\circ} \mathrm{C}\right)$} & \multicolumn{5}{|c|}{ Estabilidade $\left(15^{\circ} \mathrm{C}\right)$} & \multicolumn{5}{|c|}{ Estabilidade $\left(25^{\circ} \mathrm{C}\right)$} \\
\hline & \multicolumn{15}{|c|}{ Tempo de observação (min) } \\
\hline & 10 & 20 & 40 & 60 & 120 & 10 & 20 & 40 & 60 & 120 & 10 & 20 & 40 & 60 & 120 \\
\hline Éster metílico de óleo de soja & + & + & + & + & + & + & + & + & + & + & + & + & + & + & + \\
\hline Copolímero poliéter polimetil & + & + & + & + & + & + & + & + & + & + & + & + & + & + & + \\
\hline Ésteres de ácidos graxos com glicol & - & - & - & - & - & - & - & - & - & - & - & - & - & - & - \\
\hline Óleo mineral & + & + & + & + & + & + & + & + & + & + & + & + & + & + & + \\
\hline
\end{tabular}

(+) Estabilidade da calda; (-) Instabilidade da calda. 
Diante do relatado, pode-se concluir que a alteração da temperatura da calda influenciou as características físico-químicas de maneira diferenciada para cada adjuvante, o que demonstra a complexidade do estudo dessa relação e a impossibilidade de fazer generalizações.

O efeito dos adjuvantes nas características físico-químicas das soluções aquosas mostrouse dependente de sua composição química e formulação. O comportamento dessas características não foi semelhante mesmo para produtos com mesma indicação de uso.

A densidade não foi alterada significativamente pela variação de temperatura e adição de adjuvante à calda. A temperatura influenciou a viscosidade com maior intensidade, enquanto a tensão superficial foi a característica físico-química mais sensivel ao uso de adjuvante. O emprego de adjuvante pode levar à instabilidade da calda, requerendo maior agitação no tanque dos pulverizadores.

\section{AGRADECIMENTOS}

À Fundação de Amparo à Pesquisa do Estado de Minas Gerais (FAPEMIG), pelo suporte financeiro que permitiu o desenvolvimento deste trabalho de pesquisa.

\section{LITERATURA CITADA}

ALBERT, L. H. B.; VICTÓRIA FILHO, R. Características morfológicas da cutícula foliar e efeitos de adjuvantes no controle químico de três espécies de guanxumas. Ci. Agrotecnol., v. 26, n. 5, p. 888-899, 2002

AZEVEDO, L. A. S. Fungicidas sistêmicos: teoria e prática. São Paulo: 2001. 230 p.

BOUSE, L. F.; KIRK, I. W.; BODE, L. E. Effect of spray mixture on droplet size. Trans. ASAE, v. 33, n. 3, p. 783788, 1990.

BRONIARZ-PRESS, L. et al. The atomization of water-oil emulsions. Exper. Thermal Fluid Sci., v. 33, n. 6, p. $955-962,2009$.

CARBONARI, C. A. et al. Efeito de surfactantes e pontas de pulverização na deposição de calda de pulverização em plantas de grama-seda. Planta Daninha, v. 23, n. 4, p. $725-729,2005$.

CARLSON, K. L.; BURNSIDE, O. C. Comparative phytotoxicity of ghyphosate, SC-0224, SC-0545, and HOE-00661. Weed Sci., v 32, n. 6, p. 841-884, 1984.
CONTRERAS, O. J. G.; MUNOZ BRAVO, J. E.; FAJARDO, F. Construcción y caracterización de un tubo Ranque-Hilsch. R. Bras. Ensino Física, v. 30, n. 4, p. 4305, 2008 .

COSTA, N. V. et al. pH foliar e deposição de gotas de pulverização em plantas daninhas aquáticas: Brachiaria mutica, Brachiaria subquadripara e Panicum repens. Planta Daninha, v. 23, n. 2, p. 295-304, 2005.

CUNHA, J. P. A. R.; CARVALHO, W. P. A. Distribuição volumétrica de aplicações aéreas de agrotóxicos utilizando adjuvantes. Eng. Agric., v. 13, n. 2, p. 130-135, 2005.

CUNHA, J. P. A. R.; TEIXEIRA, M. M.; FERNANDES, H. C. Avaliação do espectro de gotas de pontas de pulverização hidráulicas utilizando a técnica da difração do raio laser. Eng. Agríc., v. 27, p. 10-15, 2007. (Numero Especial)

CUNHA, J. P. A. R. et al. Avaliação de estratégias para redução da deriva de agrotóxico em pulverizações hidráulicas. Planta Daninha, v. 21, n. 2, p. 325-332, 2003.

DOPIERALA, K.; PROCHASKA, K. The effect of molecular structure on the surface properties of selected quaternary ammonium salts. J. Colloid Interface Sci., v. 321, n. 1, p. 220-226, 2008.

DOWNER, R. A.; HALL, F. R.; THOMPSON, R. S. Temperature effects on atomization by flat-fan nozzles: implications for drift management and evidence for surfactant concentration gradients. Atomization Sprays, v. 8, p. 241-254, 1998.

GREEN, J. M.; BEESTMAN, G. B. Recently patented and commercialized formulation and adjuvant technology. Crop Protec., v. 26, n. 3, p. 320-327, 2007.

MARTINS, D. et al. Efeito de diferentes concentrações de aterbane na deposição de calda em plantas de Pistia stratiotes. Planta Daninha, v. 23, n. 2, p. 343-348, 2005.

MATUO, T; NAKAMURA, S. H.; ALMEIDA, A. Efeito de alguns adjuvantes da pulverização nas propriedades físicas do líquido. Summa Phytopathol., v. 15, n. 2, p. 163-173, 1989.

MENDONÇA, C. G.; RAETANO, C. G.; MENDONÇA, C. G. Tensão superficial estática de soluções aquosas com óleos minerais e vegetais utilizados na agricultura. Eng. Agríc., v. 27, p. $16-23,2007$. (Número Especial)

MONTÓRIO, G. A. et al. Eficiência dos surfatantes de uso agrícola na redução da tensão superficial. R. Bras. Herbic., v. 4, n. 2 , p. $8-22,2005$

MONTÓRIO, G. A.; VELINI, E. D.; MONTÓRIO, T. Definição de um coeficiente de eficácia para estudo de tensão superficial com surfactantes siliconados e não siliconados. Sci. Agr. Paranaensis, v. 3, n. 1, p. 25-34, 2004.

Planta Daninha, Viçosa-MG, v. 28, n. 3, p. 665-672, 2010 
NUYTTENS, D. et al. Effect of nozzle type, size and pressure on spray droplet characteristics. Biosyst. Eng., v. 97, n. 3 , p. $333-345,2007$.

RAMOS, H. H.; ARAÚJO, D. Preparo da calda e sua interferência na eficácia de agrotóxicos. 2006. Disponível em: <http://www.infobibos.com/Artigos/2006_3/V2/ index.htm>. Acesso em: 17 nov. 2008.

RAMSDALE, B. K.; MESSERSMITH, C. G. Nozzle, spray volume, and adjuvant effects on carfentrazone and imazamox efficacy. Weed Technol., v. 15, n. 3, p. 485-491, 2001.

RYCKAERT, B. et al. Quantitative determination of the influence of adjuvants on foliar fungicide residues. Crop Protec., v. 26, n. 10, p. 1589-1594, 2007.
SCHAMPHELEIRE, M. et al. Effects on pesticide spray drift of the physicochemical properties of the spray liquid. Precision Agric., v. 9, p. 1-12, 2008.

SINGH, M.; MACK, R. E. Effect of organosilicone-based adjuvants on herbicide efficacy. Pestic. Sci., v. 38, n. 2, p. 219-25, 1993 .

SIMITH, D. B. et al. Droplet size and leaf morphology effects on pesticide spray deposition. Trans. ASAE, v. 43, n. 2, p. $255-259,2000$.

WANAMARTA, G.; PENNER, D. Foliar absorption of herbicides. Weed Sci., v. 4, n. 1, p. 215-232, 1989. 